Slavica

bruxellensia

\section{Slavica bruxellensia}

Revue polyphonique de littérature, culture et histoire

slaves

$11 \mid 2015$

Littérature et philosophie

\title{
Entretien avec Miha Mazzini
}

Dorota Walczak-Delanois et Meta Klinar

URL : http://journals.openedition.org/slavica/1709

DOI : 10.4000/slavica.1709

ISSN : 2034-6395

Éditeur

Université libre de Bruxelles - ULB

Référence électronique

Dorota Walczak-Delanois et Meta Klinar, «Entretien avec Miha Mazzini », Slavica bruxellensia [En ligne], 11 | 2015, mis en ligne le 15 février 2015, consulté le 22 avril 2019. URL : http:// journals.openedition.org/slavica/1709; DOI : 10.4000/slavica.1709

Ce document a été généré automatiquement le 22 avril 2019.

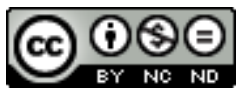

Les contenus de Slavica bruxellensia sont mis à disposition selon les termes de la Licence Creative Commons Attribution - Pas d'Utilisation Commerciale - Pas de Modification 3.0 France. 


\title{
Entretien avec Miha Mazzini
}

\author{
Dorota Walczak-Delanois et Meta Klinar
}

\section{Présentation de Miha Mazzini}

1 Né en 1961 en Slovénie (à l'époque, en Yougoslavie), Miha Mazziniest écrivain, publiciste, scénariste, réalisateur, programmateur informatique et docteur en anthropologie. Auteur de 19 œuvres littéraires, parmi lesquelles Telesni čuvaj (Garde du corps, 2000), Duhovi (Esprits, 2010), Nemška loterija (La loterie allemande, 2010), Paloma negra (2012), Polni koledarji, prazni dnevi (Calendriers pleins, jours vides, 2012), Izbrisana (La supprimée, 2014), et lauréat de prix internationaux, ses livres ont été traduits en plusieurs langues européennes.

En tant que scénariste et réalisateur, il a reçu pour son premier long-métrage le prix CIRCOM du meilleur téléfilm européen : il s'agit d'Opération Cartier, adapté de son premier roman Drobtinice (Miettes, 1986)qui, quant à lui, a battu en 1986 les records de vente des livres de l'époque dans toute la Yougoslavie. Son deuxième film, intitulé Sladke sanje (Le rêve doux, 2001) a reçu le prix national du meilleur scénario et lui a servi de base pour son troisième roman Kralj ropotajočih duhov (Le Roi des Esprits cliquetis, 2001). Son courtmétrage, Obisk (La visite, 2011) lui a valu le Prix national du meilleur court-métrage. Il est en outre membre de l'Académie européenne du cinéma.

Son troisième domaine de créativité est l'anthropologie. Dans sa thèse, il a étudié le processus de création des écrivains. Ses découvertes sont publiées dans l'ouvrage scientifique Rojeni za zgodbe (Nés pour les histoires, 2012). Cette œuvre est également accessible en anglais, au format Kindle.

Durant son temps libre, il voyage, écoute de la musique et déguste des tartes au citron. 


\section{Entretien}

\section{Premiere partie : I'homme}

Dorota walczaK: Vous êtes un homme polyvalent. Cette polyvalence permet-elle de confronter et mieux faire coïncider votre part slave avec d'autres homo sapiens?

Miha Mazzini : Qu'est-ce que c'est, cette partie slave de moi ? Cette question nous met sur un terrain extrêmement glissant, à mon avis. Si je me rappelle les romans classiques russes, par exemple: dans nombre d'entre eux, on trouve l'autodestruction et le penchant vers la souffrance à tout prix ! C'est la première chose qui me vient à l'esprit. Dans le magnifique roman Žizn' $i$ suĎba (Vie et Destin) de Vassilij S. Grossman qui a observé la lutte pour Stalingrad, il y a un paragraphe décrivant la situation suivante : les bombes tombent sur les défenseurs, il y a des cadavres partout, la ville se transforme en poussière, mais les protagonistes, eux, s'étonnent avec une joie étrange et hochent la tête : oh oh oh, mais qu'est-ce qu'ils nous font, comme ils nous détruisent, incroyable! Et si ce n'est pas l'occupant, c'est l'alcool. Nous devons nous autodétruire, d'une façon ou d'une autre. Il s'agit probablement des conséquences du système social : l'individualisation ne peut se développer que dans la démocratie, tandis que les régimes totalitaires augmentent le narcissisme que le conformisme réduit ensuite.

L'époque contemporaine est l'époque de la spécialisation: le plus apprécié, le plus respecté est celui qui en sait le plus sur un petit détail. Mais la vérité, c'est qu'il est incomparablement plus difficile d'avoir une vision large et de voir en même temps la totalité et la façon dont tout est lié.

META KLINAR : Vous surprenez sans cesse par la fraîcheur de votre perspective. D'où vient la profondeur de votre regard sur la réalité et les processus intérieurs des êtres humains? Que vous inspirent-ils?

M. M. : Comme Leonard Cohen l'a écrit et chanté : «I was born like this, I had no choice / I was born with the gift of a golden voice. " Je sais, ce que je viens de dire a l'air très arrogant. Il faut entendre la version chantée de cette chanson, qui est très « tongue in cheek ", comme le diraient les Anglais. Et surtout, vous devriez voir l'expression de mon visage en ce moment, quand je vous réponds.

Le problème "d'être ce que l'on est » réside dans le fait que l'on devient « ce que l'on est » à l'époque de la vie où on ne se rend pas encore entièrement compte de soi-même, de sa propre existence. Je parle de l'enfance précoce, de l'âge de cinq ou six ans. J'ai grandi dans un environnement où absolument tout était possible. Et puis tout s'est vraiment passé. D'un côté, cette expérience m'a laissé une éternelle incertitude, d'un autre côté, ce fait a influencé mes observations, mes réflexions et mon écriture. Entouré de mensonges, j'ai développé des méthodes de lutte pour la réalité.

м. к. : Dans votre thèse d'anthropologie vous explorez le processus de création des écrivains. Pourriez-vous nous décrire brièvement vos découvertes principales et nos erreurs les plus fréquentes sur ce sujet?

M. M.: L'image de l'auteur diffère beaucoup d'une nation à l'autre: en Grande Bretagne, on attend de lui une grande culture, de l'éducation, des débats intellectuels. En France, il porte une charge morale, la conscience de la nation. Aux Etats-Unis, il doit être aussi un entertainer. L'image de l'auteur slovène représente un alcoolique inoffensif, qui de temps en temps reçoit une aumône pour des raisons sociales.

En réalité, le nombre d'individus qui choisissent ce métier dépend de l'archétype de 
l'écrivain en collectivité. Bien sûr, en principe on se comporte de la manière que l'on attend de nous. Et la plupart du temps, on écrit de cette manière aussi, n'est-ce pas?

Être écrivain ne veut pas dire que les autres caractéristiques personnelles changent. Il y a des gens avares et généreux, certains sont introvertis, d'autres extravertis... Être écrivain veut juste dire être né avec une prédisposition, et, ce qui est le plus important, avoir appris à maîtriser son processus de création. Au moment de la création, la raison doit passer au deuxième plan, et c'est une sensation très dure, très physique. Je crois que beaucoup de gens ont peur de cette sensation et ils l'étouffent. Par conséquent, pas à pas, elle disparaît. Le premier travail créatif de chaque écrivain est donc de se créer soi-même en tant qu'auteur.

D. w. :Pourriez-vous expliquer ce que signifie pour vous la «slovénité» qui est si subtilement perceptible dans votre création littéraire?

M. M. : La société contemporaine ne jure officiellement que par l'individualisme, bien que notre époque soit beaucoup plus conforme et uniforme qu'elle ne le semble (par exemple: on a tous les mêmes voitures, téléphones, vêtements, appareils, etc.). Mais notre biologie n'a pas changé. Lorsque l'on entre dans un groupe, on s'y adapte de manière rapide et inconsciente. Même nos reflexes se coordonnent, pour ne pas mentionner les cycles menstruels. On a un nombre illimité de groupes ; on les établit au niveau du travail, de la famille, des parents, etc. Les cultures locales se forment au niveau du village ou de la ville - pourquoi donc pas aussi au niveau des états? Puisque les habitants du même état ont suivi la même éducation, qu'ils écoutent et lisent les mêmes médias... Bref, ils appartiennent au même système de valeurs et coutumes. À certains endroits, on se déchausse lorsque l'on rentre chez soi, à d'autres endroits, on ne le fait pas. Dans certaines sociétés le bien commun est quelque chose de sacré, dans d'autres, voler ce bien commun est un honneur.

La slovénité représente pour moi l'ensemble des règles non-écrites et inconscientes que chaque Slovène doit respecter s'il veut réussir dans notre société.

M. к. : Mis à part être écrivain, vous êtes aussi un observateur perspicace de la réalité de la société contemporaine. Vous nous tenez au courant de vos remarques par le biais de médias différents. Pourquoi avez-vous pris la décision d'écrire sur ce sujet?

M. M. : Parce que il n'y avait personne d'autre pour le faire. En Slovénie, un certain nombre de carrières se sont construites autour de la louange de la nation et du narcissisme. Il y a par exemple quelques livres sur l'honnêteté slovène expliquant que nous, les Slovènes, sommes la nation la plus honnête au monde. Enfant, j'ai lu ces livres et observé en même temps des employés de l'usine sidérurgique rentrer de l'usine les poches pleines de clous et autres bêtises. Leurs chefs n'étaient pas obligés de faire pareil, mais quand ils faisaient construire leurs nouvelles maisons, des camions de l'usine leur apportaient le béton.

J'ai mentionné que j'ai perçu dans ma famille l'écart entre les mensonges et la réalité. J'ai remarqué le même écart dans notre société. Nous avons un très faible contact avec la réalité en Slovénie - donc un très mauvais sens de la réalité, ce que les recherches confirment : $80 \%$ des gens estiment être d'excellents chauffeurs, au dessus de la moyenne. $90 \%$ des gens considèrent avoir une carrière merveilleuse, etc. S'exprimer d'une autre façon signifierait violer les règles de la société. Dans les petites sociétés, vous devez passer la main dans le dos et lécher les bottes très fort, car vous êtes condamné à cohabiter avec les mêmes personnes durant toute votre vie et vous ne pouvez jamais savoir si un beau jour vous n'aurez pas besoin de l'un d'entre eux. Ce 
sont des sociétés où l'hypocrisie est très hautement estimée, simplement parce qu'elle augmente les possibilités et le niveau de survie.

D. w. : En regardant vos courts-métrages, nous avons l'impression que vous jouez avec les proportions, les mesures, les distances. Vous placez l'homme dans un microcosme dont vous modifiez successivement les dimensions. Allonger le couloir, diminuer l'expression verbale, zoomer sur le visage... sont-ce là les moyens pour mieux saisir le mystère de I'homme?

M. M. : Oui, le visage... Le visage m'intéresse énormément. C'est aussi parce que j'ai commencé à aller au cinéma à l'âge de six ans, tandis que j'ai découvert le théatre beaucoup plus tard. Je n'ai jamais pu lui pardonner le fait qu'il n'offre pas la possibilité de l'enregistrement proche du visage.

Et sur le visage - les yeux. On dit qu'ils n'ont pas d'expression, que seuls les minuscules muscles qui les entourent leur donnent leur expression. Je n'y crois toujours pas.

C'est pour cette raison qu'au cours des dernières années, l'histoire courte comme forme de création m'attire particulièrement; dans tout le matériel possible, il faut trouver le visage, et sur lui, les yeux - c'est-à-dire que dans tous les mots possibles, il faut trouver une seule phrase qui exprimera l'essentiel de la personne. Dans un roman, on peut consacrer plusieurs pages à cette recherche, ce qui n'est pas possible dans une histoire courte.

\section{Deuxieme partie : les mots}

м. к : Comment transformez-vous ce qui naît à l'intérieur de vous et qui est indescriptible (et que l'on nomme « inspiration ») en mots?

M. M : Il est surprenant de voir à quel point le processus de création est physique. Il vous laisse une fatigue physique très forte, malgré le fait que l'on soit «juste assis » pendant une ou deux heures. J'ai scanné mon cerveau à des moments différents: lorsque que je ne faisais rien; lorsque je lisais quelque chose qui ne me plaisait pas; lorsque je lisais quelque chose qui me plaisait ; lorsque j'écrivais. Pendant le processus de création, l'irrigation sanguine augmente dans la totalité du cerveau, puisqu'en imagination nous bougeons, parlons, sentons d'une manière très intense, ce qui est très rare dans la vie réelle. Et qui est plutôt désagréable en réalité. Je ne suis pas une drama queen dans ma vie réelle, je le suis dans l'espace de l'imagination, à l'intérieur de mon crâne.

Bref, c'est difficile. Comme toutes les choses les plus belles qui peuvent nous arriver dans la vie.

D. $\mathbf{w}$ : La littérature est-elle pour vous aussi une affaire d'être ? Plus d'être, plus de paraître ?

M. M. : Affaire d'être ? Leonard De Vinci a réfléchi sur ce sujet et a constaté : tout ce qui reste à l'homme, ce qui n'est pas créatif, c'est travailler sur le champ, se nourrir et chier. C'est-à-dire que la vie humaine sans création égale celle du bétail. Je dirais que la création permet une qualité de vie supérieure. Et ce qui compte le plus à cet égard, c'est le sentiment de sa propre créativité, quel que soit le domaine de création : peu importe que l'on ait écrit un roman ou tricoté un pull.

D. w. : J'aimerais faire un point sur l'aspect fort poétique de votre œuvre. Vous sentez-vous appartenir à la génération nourrie par la poésie du grand Tomaž Šalamun ? La poésie est- 
elle pour vous une catégorie esthétique, le domaine des paroles, une autre façon de raconter? D'ailleurs, avez-vous déjà écrit des poèmes ?

M. M. : Non. Bien sûr, j'ai écrit des poèmes étant adolescent, mais ensuite, j'en ai terminé avec la poésie. Pour l'instant, elle ne m'attire pas du tout. Peut-être est-ce ainsi parce que je suis " un homme de structure » : les histoires, les romans, les scénarios se composent dans ma tête comme un réseau, comme des liaisons de symboles, de sens et de fonctions. Ensuite, ces liaisons trouvent un équilibre et forment un système qui me plaît (ce qui ne veut pas dire que je vais le décrire d'une manière satisfaisante).

J'ai lu Šalamun en tant que gamin, j'ai en fait lu énormément de poésie. Mais plus tard, quand j'ai commencé à apprendre comment écrire les histoires, j'ai passé mon apprentissage à écouter de la musique country. Sérieusement: dans ce genre de musique, vous avez deux minutes et demi pour raconter toute l'histoire. Je possédais quelques disques et je les ai rendus lisses à force de les écouter, en bougeant les phrases, en cherchant les améliorations, en cherchant des réponses. Dieu merci, j'ai trouvé Tom T. Halle, par exemple - c'était par pure coïncidence (synchronisée, aurait ajouté Jung).

м. к. : Les thèmes les plus fréquents de vos œuvres littéraires et cinématographiques sont : la peur de prendre une décision; la (non)existence de dieu; le vide de la vie et/ou la recherche de son sens; le poigs qui peut peser sur un enfant (qui peut être déjà adulte) à cause du comportement anormal de ses parents. Êtes-vous d'accord? Estimez-vous qu'au fond il s'agit d'un seul thème : la recherche d'identité ?

M. M. : L'identité est sans aucun doute un élément important, et c'est valable pour toute la société occidentale. Si on ne se représente plus la foule comme le corps allongé de dieu ou d'un souverain, quand la société se met à se décomposer en individus - ce qui a commencé à la Renaissance - dans ce cas, l'individu doit se poser la question : et MOI, qui suis-je ? La contemporanéité est le mélange de toutes les réponses possibles et imaginables à cette question.

Ce qui est paradoxal, c'est que si vous recevez la réponse à votre question sur l'identité de la part des autres, cela signifie que vous faites partie de la foule. Si vous trouvez la réponse tout seul, vous êtes vous-même.

D. w. : II y a quelques années, en 2010, vous avez publié une histoire courte, Organizem (L'organisme; en anglais That Winter), qui vous a valu le prix Pushcart aux Etats-Unis. Le sujet de cette histoire ainsi que de certaines autres de vos histoires courtes est la guerre. Les mots aident-ils ou dérangent-ils dans la transmission de l'expérience irracontable et effroyable?

M. M. : L'écrivain est toujours aussi un témoin. Le témoin de l'expérience humaine générale, de son époque et du destin individuel. Rappelons-nous les témoignages des camps de concentration : quelqu'un doit survivre et décrire ce phénomène pour que les futures générations sachent comment on a vécu certaines épreuves. Je suis entré dans une période de la vie où les situations suivantes commencent à se produire : des gens qui, lorsqu'ils avaient vingt ans, faisaient la grimace, s'exclamant : "Oh, s'il vous plaît, quelle bêtise, écrire! Ce qui compte, c'est la carrière, l'entreprise, la richesse ! Ces gens viennent maintenant me voir en me demandant: "S’il te plaît... écris mon histoire! C'est la seule chose qui reste au moins une génération ou deux. » Et qu'est-ce que je leur réponds? «Allez vous faire... » 


\section{Troisieme partie : l'image}

\section{D. $\mathbf{w}$. : Comment l'image naît-elle en vous et dans votre œuvre?}

M. M. : Parfois c'est une image, parfois une phrase ou une odeur ; toujours, c'est une sensation nouvelle, spéciale et unique. Quelque part derrière le cou, en bas du ventre. C'est une sensation forte et exceptionnelle, lorsque l'on sent que le processus de création vient de commencer. Une sensation qui rend les auteurs dépendants et les jette dans des doutes profonds si elle n'apparait pas pendant longtemps.

D. w. : Dans votre film Sladke sanje (Le rêve doux, 2001) il y a une part importante occupée par l'impondérable - la partie impossible du destin. Vous aimez le thème du hasard, de l'impossible, de la dérision?

M. M. : Je vous invite à lire sur Wikipedia la description de la démarche scientifique de Benjamin Libet : au moment où nous prenons la décision de bouger la main, les parties inconscientes de notre cerveau sont au courant de cette décision plus tôt que notre raison. Ensuite, lisez la description du fait que la raison est toujours en décalage d'une demi-seconde avec la réalité. Pensez-y. Imaginez-vous cela. On contrôle incomparablement moins de ce que l'on s'imagine.

Sladke sanje est en partie un film sur mon enfance, où, comme je l'ai dit auparavant, régnait l'imprévisibilité.

\section{Quatrieme partie : l'univers}

D. w. : Le lieu de naissance est-il important pour vous ? Si vous pouviez changer votre lieu de naissance, quel lieu choisiriez-vous et pourquoi?

M. M. : Bien sûr qu'il est important. Encore plus pour l'écrivain, car il est né dans une langue, et si cette langue a peu de lecteurs et surtout peu d'acheteurs de livres, la conséquence est que toute sa vie, cet écrivain pourra écrire seulement dans ses rares heures libres. En d'autres mots, il essaiera de courir en tant que coureur professionnel dans les conditions et à partir du point de départ d'un amateur.

м. к. : On trouve sur votre site $^{2}$ un grand nombre de conseils concrets pour les nouveaux écrivains, pas encore reconnus : comment présenter une partie de son œuvre, ou comment et à quelle fréquence chercher à contacter les éditeurs, etc. Votre altruisme est un geste chaleureux. Qu'est-ce qui vous a poussé à le faire? Tant de gens vous ont-ils harcelé avec les mêmes questions, ou avez-vous simplement un cœur d'or?

M. M. : À mon avis, chaque homme devrait régulièrement transférer au moins un certain pourcentage de ses revenus à des œuvres de charité et consacrer au moins quelques heures par semaine au bien commun. Et oui, je recevais et je reçois toujours beaucoup de questions à ce sujet. Quand je me suis rappelé des difficultés que j'ai éprouvées lors de mes recherches d'informations pertinentes, j'ai décidé d'écrire une courte introduction à ce sujet. Au moment où je reçois une question qui ne figure pas dans mon introduction, je l'ajoute et complète le texte sur mon site.

D. w. : Il y a beaucoup d'espaces fermés dans votre œuvre : la chambre d'hôpital en est un. $Y$ voyez-vous un lien avec le teatrum contemporain, la scène d'un drame important?

M. M. : Non. Le théâtre est pour moi un parfait inconnu. Au cours des dernières années, j'ai intensivement cherché à comprendre comment écrire un drame.

En ce qui concerne les scénarios de films, il faut savoir qu'il s'agit d'une écriture qui doit respecter des contraintes financières. Par exemple : si vous n'avez pas de budget, 
de jolies blondes ne courront pas sur une plage hawaïenne, à la place de cela, deux types discuteront dans une chambre.

м. к. : Croyez-vous que la littérature - et l'art au sens large - puisse changer le monde ?

M. M. : Chacun de nous marche avec son minuscule grain de sable jusqu'au sommet de la montagne. Il y apporte sa contribution, peut-être pas plus grande qu'une poussière. On doit accumuler beaucoup de grains avant que l'avalanche ne se déclenche, avant que le terrain ne change. Personne ne sait quel grain le fera, c'est pourquoi on continue.

D. w. : Je vais répéter ma question posée lors de notre rencontre à l'ULB en 2014 avec les étudiants. Vous avez aimé cette question et vous m'avez dit que vous deviez réfléchir avant d'y répondre. Je n'ai pas oublié cette question, donc: qui serait votre Béatrice, votre guide dans l'enfer et comment l'imaginez-vous, cet enfer?

M. M. : L'enfer? Faire partie de la foule dans un centre commercial. Donc un espace sans Béatrice.

D. w. : Et comment vous imaginez-vous le paradis?

M. M. : Le paradis ?

« Heaven, I'm in heaven / And my heart beats so that I can hardly speak. » Intéressant, en ce moment je suis justement en train d'apprendre à jouer cette chanson sur un banjo. Ce serait le paradis, si j'avais l'oreille musicale. Et bien sûr : « And I seem to find the happiness I seek / When we're out together dancing cheek to cheek ».

Au nom de toute l'équipe de Slavica Bruxellensia nous vous remercions pour cet entretien.

\section{NOTES}

1. Tomaž Šalamun (Zagreb1941 - Ljubljana 2014), poète slovène contemporain et un des piliers fondamentaux du modernisme slovène. Il est considéré comme l'un des grands poètes de l'Europe centrale après la Deuxième Guerre mondiale. Il a publié plus de 40 livres de poésie qui ont été traduits dans plus de 20 langues. Il s'est bâti une réputation internationale autour de ce qu'un critique a appelé « la complexe santé mentale des limites » en décrivant son esprit anarchique subtil. Sa poésie touche à la politique aussi bien qu'aux expériences intimes. Elle symbolise l'ampleur infinie du modernisme et montre que le monde spirituel est sans limites.

Il a été membre associé de l'Académie slovène des Sciences et des Arts et lauréat de plusieurs prix internationaux de poésie. La nouvelle génération des poètes slovènes s'inspire de son écriture.

2. http ://www.mihamazzini.com/EN/ 
INDEX

Index chronologique : XXe siècle

Mots-clés : littérature slovène

Index géographique : Slovénie

\section{AUTEURS}

\section{DOROTA WALCZAK-DELANOIS}

Responsable de la Chaire de Polonais, section de Langues et Littératures modernes, option Slaves, de l’Université Libre de Bruxelles (Belgique) ; Rédactrice en chef de Slavica Bruxellensia

META KLINAR

Lectrice de slovène, Université Libre de Bruxelles 